\title{
Advantage of HR-STEM for Evaluating Ultra-fine Carbides Embedded in Steel
}

\author{
Katsumi Yamada ${ }^{1}$, Haruo Nakamichi ${ }^{2}$ and Kaoru Sato ${ }^{3}$ \\ 1. JFE Steel Corporation 1-1, Minamiwatarida-cho, Kawasaki-ku Kawasaki, 210-0855 Japan \\ 2. JFE Steel Corporation 1, Kokan-cho, Fukuyama, 721-8510 Japan \\ 3. JFE Steel Corporation 1, Kawasaki-cho, Chiba, 260-0835 Japan
}

Achieving homogeneous distribution of fine carbides is key technology for obtaining high strength steels with excellent formability. Some kind of carbides such as typical MC or M23C6 represents interphase precipitation in a well-controlled hot rolling process as typically exemplified in Fig.1. Continuous and periodic row corresponds to one sheet of precipitates. In addition to this type of precipitation, there are also three equivalent platelet carbides in ferrite matrix with well-known Baker Nutting relationship. For both type of precipitation, characterization of them has been conducted by various techniques such as chemical analysis for extracted residue and electron microscopy in order to optimize steel composition and process condition of the steel. Although conventional TEM analysis is useful to clarify its size, morphology and components simultaneously, it is still difficult to conduct accurate observation and an elemental analysis of single nm-sized MC type carbides embedded in ferrite matrix of a recent developed high strength steel ${ }^{(1)}$. Generally, MC type carbide such as $\mathrm{TiC}$ or $\mathrm{NbC}$ in ferrite is crystallographic semi-coherent and its effective volume fraction is too small for both imaging and analysis. STEM-HAADF has been widely used in a field of material science because it makes possible imaging with atomic resolution and high performance analysis using sub-nm electron probe, but for single nm-sized carbide HAADF is not superior to a conventional TEM because its $\mathrm{Z}$ contrast is usually lower than that of surrounding ferrite matrix.

In this study, conventional STEM-BF and ADF, which are strongly affected by diffraction or strain contrast as TEM, were applied for clear visualizing and elemental analysis of individual fine carbide. High throughput analysis using proper STEM condition was established for promoting studying advanced steel with dispersion strengthening. Commercial $800 \mathrm{MPa}$ grade Ti-Mo bearing hot rolled steel was investigated using probe Cs-corrected STEM (FEI TITAN80-300) at 300kV acceleration voltage. Specimens for HRSTEM experiments were prepared by FIB and elemental analysis was conducted using conventional SSD detector. Fig. 2 shows STEM-ADF image series from [001] bcc direction by changing minimum collection semi-angle $(\beta)$ to an annular detector. This field of view contains three equivalent variant of Ti-Mo complex MC carbide. As indicated by arrows in Fig.2 (d), platelet MC parallel to the specimen surface with several $\mathrm{nm}$ in side length became clear by decreasing $\beta$ as well as strong dislocation contrast while that visibility was insufficient at ordinary HAADF condition. Very thin cross sectional $\mathrm{MC}$ was also visible with lattice resolution as shown in Fig.3. It was found that optimum range of lower collection semi angle for ideal ADF imaging was from $18 \sim 28 \mathrm{mrad}$. Visibility of these MC by using conventional BF-STEM was also high. Under keeping high resolution STEM image, required probe current for EDS analysis was investigated. Although normal SSD used in this experiment was inferior to recent high efficiency SDD, around 300pA was enough to obtain statistically sufficient EDS spectra and lattice resolution STEM images simultaneously. 
Improvement in visibility of ultra fine platelet MC with proper ADF or BF-STEM imaging is certainly due to diffraction contrast of itself. Especially, parallel variant of MC shown in Fig. (c) and (d) shows subtle bright edge contrast and this is probably due to strain contrast in surrounding matrix.

Sub-nano analysis with Cs-corrected fine probe has made clear that MC has almost uniform composition, $\mathrm{Ti} / \mathrm{Mo}=1$ along its thickness direction. There is not clear core-shell structure as reported in other materials such like aged-Al alloy ${ }^{(2)}$. This indicates that no elemental selection at a nucleation stage of these $\mathrm{MC}$, the composition of carbide former is almost proportional to the balance of steel composition. It can be concluded that ordinary STEM was useful for both visualizing single nm-sized $\mathrm{MC}$ and conducting sub-nano analysis and made clear its nucleation stage in practical high strength steel.

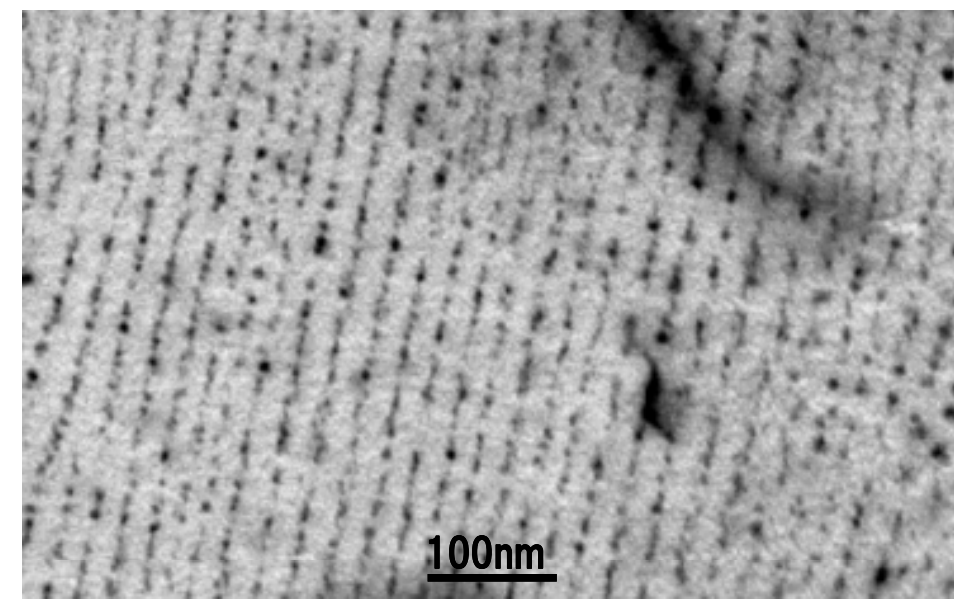

Fig.1 Typical STEM-BF image of inter-phase precipitation of Ti-Mo complex carbide in a high strength hot rolled steel sheet.
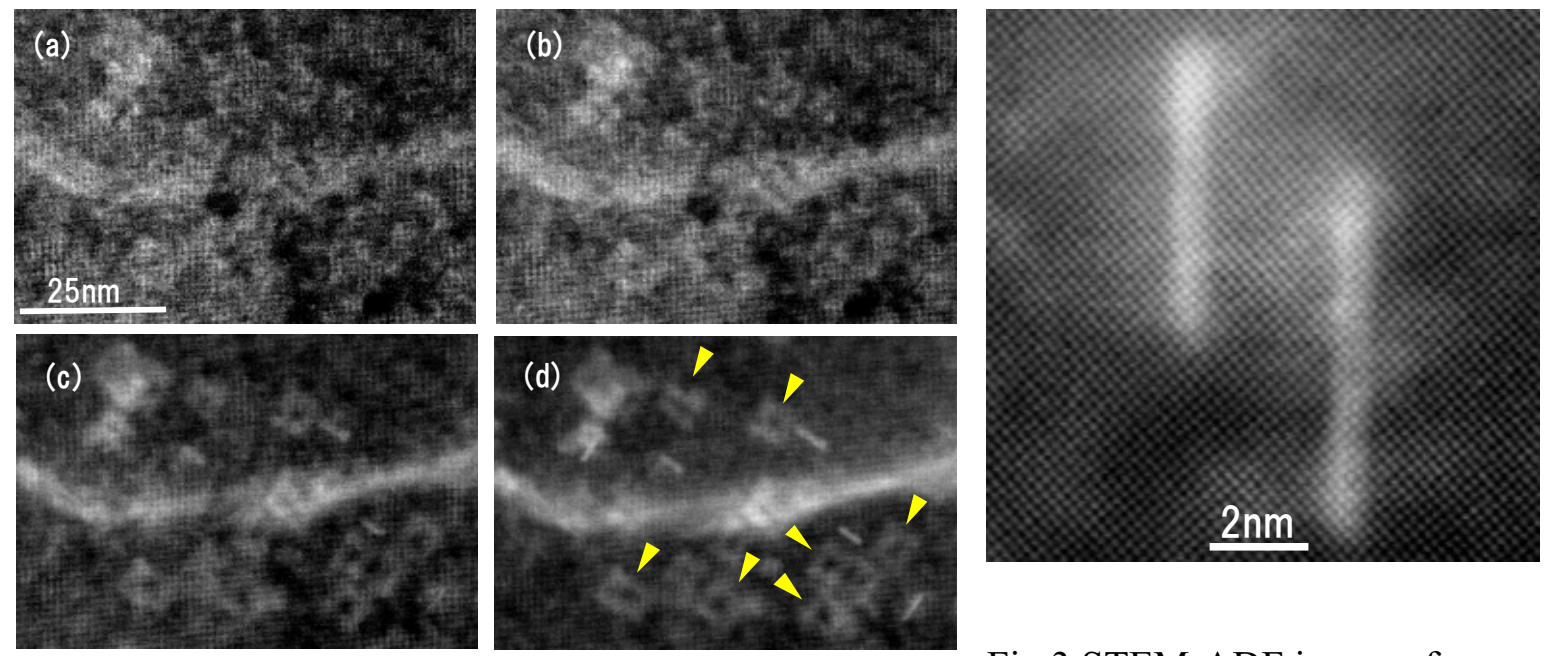

Fig.2 STEM-ADF image series from $[001]_{\text {bcc }}$ direction by changing collection semi-angle.

\section{References:}

[1] Y. Funakawa et al, ISIJ Int. 2004; 44: 37.

[2] V. Radmillovie et al., Proceedings of $14^{\text {th }}$ European Microscopy Congress (2008) vol.2, p473-474. 